\title{
Jovian impact flashes and their implication to small bodies
}

\author{
Junichi Watanabe \\ National Astronomical Observatory of Japan \\ e-mail: jun.watanabe@nao.ac.jp
}

\begin{abstract}
Optical flashes on the surface of Jupiter were observed by amateur astronomers in June and August 2010. It is thought that these phenomena were bright meteors caused by the collision of small celestial bodies of a few to $10-\mathrm{m}$, and that they seemed to be more frequent than expected. If the frequency and the scale of these phenomena are investigated, the size distribution down to size of a few $\mathrm{m}$ can be decided at around the giant planet region. If the systematic observation is achieved, it will be a unique attempt to use the giant planets as a natural detector of small bodies.
\end{abstract}

Optical flashes on the surface of Jupiter were observed by amateur astronomers in June and August 2010. Four amateur astronomers in Japan recorded a flash at $3 \mathrm{~h} 22 \mathrm{~m} 12 \mathrm{~s}$ on August 21 (UT). The coordinate is 140.4 and +21.1 degrees in system II. The duration was about two seconds, and the brightness was 6.2 magnitudes. The preliminary light curve is analyzed. This is presumed to be an equivalent or slightly smaller scale than that in June(Hueso et al. 2010). It is thought that these phenomena were bright meteors caused by the collision of small celestial bodies of a few to $10 \mathrm{~m}$, and that they seemed to be more frequent than expected. If the frequency and the scale of these phenomena are investigated, the size distribution down to size of a few $\mathrm{m}$ can be decided at around the giant planet region. In case of Earth, the brightness of meteors depends not only on sizes but also on the entry velocity. However, in the case of Jupiter, the entry velocity becomes almost similar value $(60-64 \mathrm{~km}$ per second) which is almost independent on the direction of the orbits of bodies because of the strong gravity of Jupiter. We do not have any uncertainty for estimating size of impacting bodies from the brightness of the flashes. On the other hand, we have large uncertainty in the size distribution of small bodies in the giant planet region, because we cannot see directly any bodies of less than $1 \mathrm{~km}$. Therefore, if the systematic observation is achieved, it will be a unique attempt to use the giant planets as a natural detector of small bodies.

\section{Reference}

Hueso, R., et al., 2010, ApJ 721, L129-L133 